\title{
The My-T study: Patient satisfaction and preference comparing topical and nasal testosterone therapies
}

Jay Lee, $\mathrm{MD}^{1}$; Gerald Brock, $\mathrm{MD}^{2}$; Jack Barkin, $\mathrm{MD}^{3}$; Nathan Bryson, $\mathrm{PhD}^{4}$; Matthew A. Gronski, $\mathrm{PhD}^{4}$; Ross Ormsby, $\mathrm{PhD}^{4}$

${ }^{1}$ Department of Surgery, Division of Urology, University of Calgary, Calgary, AB, Canada; ${ }^{2}$ Department of Surgery, Division of Urology, St. Joseph's Health Care, London, ON, Canada; ${ }^{3}$ Department of Surgery, University of Toronto, Humber River Regional Hospital, Toronto, ON, Canada; ${ }^{4}$ Acerus Pharmaceuticals Corporation, Mississauga, ON, Canada

Acknowledgements: Editorial assistance in the preparation of this manuscript was provided by Christina Sanguinetti.

Cite as: Can Urol Assoc J 2019 February 26; Epub ahead of print. http://dx.doi.org/10.5489/cuaj.5680

Published online February 26, 2019

$* * *$

\section{Abstract}

Introduction: Natesto ${ }^{\circledR}$, testosterone nasal gel (TNG) is an intranasal testosterone therapy (TTh) used to restore testosterone levels and improve symptoms of hypogonadism. Treatment requires application two (bid) or three (tid) times daily. The Treatment Satisfaction Questionnaire for Medication (TSQM) and a Patient Preference and Use (PPU) Questionnaire were used to obtain patient feedback on the use of TNG and compare to experience with topical TTh.

Methods: The study enrolled 24 TTh-naive (TThN) and 93 TTh-experienced (TThE)

hypogonadal men. Treatment lasted up to 120 days, with titration at day 90 to determine the most appropriate dose for restoration of testosterone levels (11 mg bid or tid). Patient satisfaction and symptom changes were measured at days 0, 30, 60, 90, and 120. The PPU Questionnaire was performed at study entry and study completion.

Results: Symptoms improved from baseline (30.6) to day 90 (35.1) $(\mathrm{p}<0.0001 ;+15 \%)$, consistent with testosterone replacement. TNG increased scores for effectiveness $(+20 \%)$, convenience (+30\%), and global satisfaction (+3\%) as compared to their previous topical TTh. TThE patients reported ease of use, convenience, efficacy/effectiveness, and travel friendliness as "likes" of TNG therapy. Overall, 67.2\% of patients agreed or strongly agreed that they preferred TNG over topical TTh and 59\% sought a prescription to continue treatment with TNG. 
Conclusions: Patients switching from topical TTh to TNG reported significant improvements in symptoms and patient satisfaction compared to their previous topical TTh. Patients also reported a significant improvement in convenience with TNG despite two to three times daily application. Preference, satisfaction, and convenience may translate to better treatment compliance.

\section{Introduction}

A variety of testosterone replacement therapy (TTh) options are available to patients to treat hypogonadism. These include intramuscular injections, topical gels and patches, buccal patches, nasal gel, and in some countries oral capsules. ${ }^{1}$ Patients and physicians weigh the advantages and disadvantages of each when making a choice that best fits the therapeutic needs, preferences, safety/tolerances and lifestyle. Factors include convenience, cost, potential adverse local (irritation) or systemic (cardiovascular, hematocrit) reactions, transference, administration, smell/odor, and physician recommendations. ${ }^{2,3,4,5,6,7,8}$

Nasal delivery, with Natesto ${ }^{\circledR}$ (TNG) $4.5 \%$ testosterone nasal gel, is a new and unique route of administering testosterone. ${ }^{9}$ TNG provides a peak-and-trough (ultradian) serum profile of testosterone, which, like the natural circadian rhythm of endogenous $\mathrm{T}$ production, is not steady state. ${ }^{10,11,12}$ The resulting serum profile provided by bid dosing from the metered-dose pump dispenser (Fig 1) is believed to provide a unique combination of advantages related to safety and efficacy, including: (i) no touch administration that avoids transference; (ii) fast/easy self-administration requiring only about 10 seconds per dose; (iii) very low risk of experiencing supraphysiological $\mathrm{T}$ even at the maximum recommended daily dose due to a divided dose ${ }^{13}$; and, (iv) retention of gonadotropin feedback with potential retention of sperm count and the possibility of lessening of testicular atrophy. ${ }^{14}$ Perceived disadvantages of TNG may include (i) repeat dosing and (ii) the nasal route of administration.

The My-T study was designed to address two questions related to treatment with TNG: (1) Given the proven safety of the maximum recommended daily dose, can titration be performed based on symptom improvement, using total testosterone (TT) levels after dose adjustment to confirm restoration of levels for safety and efficacy? (2) What are patients' perceptions of dosing with TNG relative to more widely prescribed topical TTh medication? In this paper we present the results of the second question.

This study primarily recruited hypogonadal males who were taking a topical TTh medication for hypogonadism and were willing to switch for a period of up to 120 days. Patient satisfaction data was captured just prior to TNG initiation (baseline) for TThE patients and compared to the results after completing the treatment period in order to provide quantitative and qualitative information on efficacy, effectiveness, satisfaction and preferences. 


\section{Methods}

\section{Study population}

Patients were adult hypogonadal males ( $<65$ years of age) with documented TT levels $\leq 10.4 \mathrm{nmol} / \mathrm{L}$. Approximately, $75 \%$ of patients enrolled were receiving treatment with a topical TTh for at least 3 months prior to the study and were on active treatment at the time of inclusion. Approximately, 25\% of patients were treatment-naïve.

\section{Study design}

The My-T Study (NCT02937740) was a multicenter, single-arm intervention study treating hypogonadal males with TNG for up to 120 days with potential dose adjustment at Day 90. This study was conducted under CTA issued by Health Canada (Sept 15, 2016), and in accordance with guidelines set forth by the International Conference on Harmonisation (ICH) Guidelines for Good Clinical Practice (GCP) and in accordance with ethical principles that have their origins in the Declaration of Helsinki regarding treatment of human patients in a study.

The study assessed patients’ symptoms using the Quantitative Androgen Deficiency for Aging Males Questionnaire (qADAM) ${ }^{15}$ and measured patient treatment satisfaction using the Treatment Satisfaction Questionnaire for Medication (TSQM). The TSQM Version 9 is a 9-item validated instrument with domains for Effectiveness (3 items), Convenience (3 items), and Global Satisfaction (3 items). ${ }^{16}$ Domain scores are the sum of values of questions in each domain expressed as a percentage of the maximal possible score. The TSQM was administered on Days 0, 30, 60, 90 and Day 120 and analyses were performed using ANOVA (analysis of variance). Missing data points were assessed using the Last Observation Carried Forward (LOCF) methodology and statistical analyses, as deemed appropriate. The primary efficacy analyses were performed using the intent-to-treat (ITT) LOCF population. Changes in TSQM domains were analyzed using a Shapiro-Wilk normality test. Based on results, p-values were calculated using a paired t-test (2-sided) for normally distributed data or a Wilcoxon signed-rank test (2-sided) for non-normally distributed data. All patients completed an internally-developed PPU Questionnaire. The questionnaire asked patients to designate a degree of importance (from very important to not important at all), agreement (strongly agree to strongly disagree), asked qualitative questions about patients' likes and dislikes, and recorded their willingness to switch to TNG, which was confirmed by phone 30 days after completion of the treatment period. Normality of patient response data was determined using the Shapiro-Wilk normality test. pvalues were calculated using paired t-test (2-sided) for normally distributed data or Wilcoxon matched pairs signed-rank test (2-sided) for non-normally distributed data. Analyses were performed on the complete study population and stratified by dosage or prior TTh experience. pvalues were not adjusted for multiple comparisons. 


\section{Results}

Table 1 provides the demographic information for the 117 patients who were enrolled from 11 Canadian sites. Age, weight, height, age at diagnosis and racial breakdown were similar between TTh-naïve (TThN) and TTh-experienced (TThE) patients. Of these, 93 (79.5\%) were on topical testosterone treatment for at least the previous three months; 24 patients (20.5\%) were TThN. All subjects received the starting dose of $22.0 \mathrm{mg}$ daily (11 mg bid). The dose was adjusted, as necessary, at Day 90 to manage symptoms. Seventy-seven percent $(\mathrm{N}=77)$ of patients were in the normal TT range at study completion with a mean serum TT of $19.4 \mathrm{nmol} / \mathrm{L}$. Seventy (70; 70\%) patients stayed on the $22.0 \mathrm{mg}$ dose, while 30 (30\%) were uptitrated to the $33.0 \mathrm{mg}$ dose. Seventy-eight (78) patients (67\%) completed the study in its entirety. Eighteen (18) patients withdrew consent, 5 patients withdrew consent due to an AE, 10 patients were lost to follow-up, 5 patients were discontinued due to an adverse reaction and 1 patient withdrew for other reasons. One hundred patients (100; 85\%) were included in the ITT population, including 77 (77.0\%) TThE patients.

Table 2 provides absolute values for TSQM domain responses for all patients. For TThE patients $(\mathrm{N}=69)$, TSQM was given at Day 0 and represented patient assessment of the topical medication at study initiation as a reference point for comparison. TThN patients, who were not on active treatment, were not administered the TSQM at Day 0. In general, there was an increase in all domains of treatment satisfaction, which was the strongest between baseline (Day 0) and Day 30, the first follow up visit after starting TNG. The mean values for all 3 TSQM domains from Day 30 to study endpoint were comparable between TThE and TThN patients, indicating that both groups reported similar TNG treatment satisfaction.

Tables 3 and 4 provide a summary of changes from baseline for each of the TSQM domains for patients stratified by dose at study completion. Of the $60 \mathrm{TThE}$ patients who completed the study at the bid dose, there were significant increases from baseline in the Effectiveness (+9.6 [SD 25.9]; $\mathrm{p}=0.020)$ and Convenience Domains (+18.9 [SD 21.4]; $\mathrm{p}<0.0001)$ at study conclusion, but significance was not achieved for Global Satisfaction (-0.6 [33.0]; $\mathrm{p}=0.8966$ ) (Table 3). For TThE patients completing the study at the tid dose $(n=15)$, there was a statistically significant ( $\mathrm{p}=0.044$ ) increase from baseline in the Effectiveness Domain from 37.3 (SD 18.1) to 50.0 (SD 23.3) at Day 90 while receiving the bid dose and another increase to 58.9 (SD 15.7) on Day 120 after uptitration to tid dosing. Similarly, the Convenience Domain showed a significant increase ( $\mathrm{p}=0.0049$ ) from 56.9 (SD 22.7) at baseline to 76.8 (SD 16.7) through Day 90, which was followed by a modest increase to 78.1 (SD 16.3) at Day 120. The statistically significant ( $\mathrm{p}=0.0252$ ) increase from baseline in the Global Satisfaction Domain from 40.8 (SD 19.4) at baseline to 53.4 (SD 25.3) at Day 90 remained essentially unchanged (52.9 [SD 21.9]) at Day 120.

Patients were asked about their most important symptom in a multiple choice question on the PPU Questionnaire ( $\mathrm{N}=100)$. There was no significant difference in responses when stratified 
by prior TTh experience (data not shown). Results of the combined population are shown in Table 4. Patients reported difficulty achieving/maintaining an erection (28.3\%), fatigue/loss of energy (25.0\%) and sex drive (25.0\%) as the most important symptoms to treat.

Patients were also asked to provide a list of both what they "liked" and "disliked" about TNG. Patients' "likes" that occurred most frequently included: ease of use ( $>45$ responses), convenience (12), efficacy (10) or effectiveness and travel friendliness (10). "Dislikes" that patients reported 10 or more times included: nasal drip (17), feeling/discomfort (12) and smell (10). Rarely did patients indicate "frequency of use" as a dislike (1 response); and, this was not reported as a reason for discontinuation. The relative importance of likes and dislikes, or how this impacted choice or preference, was not probed directly; yet, 64\% of all TThE patients agreed/ strongly agreed that they preferred nasal testosterone over topical medication. Also, 30 days after completing the study treatment, a follow-up call determined that $59 \%$ of patients sought a prescription in order to continue medicating with TNG, while $22 \%$ of patients were still considering it.

\section{Discussion}

Hypogonadal patients who seek TTh have a wide choice of medical preparations for treatment including pellets, intramuscular injections, topical, patch, oral, buccal and nasal. In general, all of these preparations are considered safe and effective. While independent surveys show fairly good overall rates of satisfaction ( $>70 \%$ patients), ${ }^{17}$ only $18.63 \%$ of first time TTh users in the United States refilled their prescription within three months. ${ }^{18}$ Given the various treatment options, it is perhaps central to better understand what factors are important to patients when selecting one option over another, as well as issues that may impact patient preference and patient compliance.

Patient reported outcomes (PRO) or Questionnaires have been utilized to improve treatment paradigms. ${ }^{19,20}$ There is evidence suggesting that integrating PROs in clinical settings improves communication between patients and clinicians allowing for positive effects on patient care, outcomes and compliance. ${ }^{21}$ The ability to evaluate and document changes in symptoms and patient satisfaction is of significant clinical value, specifically when it comes down to patient choice and preference, therefore, contributing to a higher quality of care. ${ }^{22}$ Quantification of treatment satisfaction with the TSQM is a pertinent, validated and reliable tool to understand and compare treatment modalities. In prior studies, TSQM correlated with patient compliance and medication adherence with good consistency. $23,24,25$

The majority of patients (75\%) enrolled in this study had prior topical TTh experience and were able to compare their prior treatment to the nasal delivery form of TTh using the TSQM. A comparison of dosing recommendations for TNG and topical preparations is shown in Table 5.

While TThE patients were modestly satisfied with their topical medication, as shown by TSQM and qADAM scores at study entry, qADAM domains (particularly energy level, libido 
and strength/endurance) as well as the TSQM domain for Effectiveness and Convenience (p $<0.05$ ) were all statistically higher at study completion. Overall, qADAM and TSQM results suggest that TNG is effective in achieving patients' primary goal of therapy and symptom relief. TThE patients also found TNG to be significantly more convenient compared to their prior topical medication. It is noteworthy that an increase in daily dose from bid to tid did not negatively impact patient responses in either the Effectiveness or Convenience domains. Lastly, while TThN patients did not have prior experience with TTh at baseline, mean endpoint values in each of the TSQM domains were comparable to those of experienced patients, indicating that prior exposure to a topical testosterone product is not necessary to observe satisfaction with TNG.

Possible weaknesses in the study include a small subset of naïve subjects in the trial, which did not allow complete statistical analysis of this group. The main goal of this analysis was to provide a comparison of nasal and topical medications, which is a perspective that naïve patients lack. Yet, endpoints for the TThN subset were similar to the larger populations, suggesting that satisfaction with TNG and preferences are similar for all patients. The discontinuation rate in this study was greater than observed in prior experiences with TNG, which may have affected the quantitative results reported, yet, there remains a significant proportion of subjects who completed the study and who clearly perceived benefits from access to TNG. The PPU Questionnaire was not a validated instrument and used multiple choice and open-ended questions that provide useful feedback, which was not quantified, but rather provided indicators. There may be a bias in terms of patient selection in the TThE patient group, as some patients may have been 'unhappy' with their previous topical TTh prior to entering this study. Patients who were very satisfied with their topical TTh may have been less likely to enter the study. However, the baseline qADAM mean was $>30$ indicating that patients in this study felt their condition at an average level of symptom relief on topical TTh, while the mean baseline TSQM convenience scores were over 50 suggesting patients found the topical product to be "somewhat easy" to use.

\section{Conclusion}

TNG restored testosterone levels to normal range and improved symptoms for patients in this study. For patients switching from a topical medication to TNG, effectiveness, convenience and symptoms all demonstrated significant improvements indicating that the divided dose, ultradian profile with TNG is at least, and possibly more, efficacious than the steady state profile provided by topical testosterone. When followed-up 30 days post-treatment, the majority of patients in this study reported having made the switch to TNG as their preferred choice of medication for testosterone replacement.

Furthermore, patient satisfaction improved significantly on TNG relative to a topical TTh. The Convenience domain of the TSQM showed the strongest improvements. Patients overwhelmingly liked the "ease of use/application” of TNG (the most frequent response to any 
like or dislike). Convenience was not impacted by increasing dose frequency from bid to tid. Repeat dosing, which can, at times, be a compliance risk, was actually preferred in this study suggesting that TNG's 10-second, no touch application method is a real and significant improvement relative to the, up to, 20 minute once-daily application of a topical gel. 
Figures and Tables

Table 1. Summary of demographic data (ITT analysis)

\begin{tabular}{|l|c|c|c|}
\hline & \multicolumn{3}{|c|}{ ITT } \\
\hline Parameter & $\begin{array}{c}\text { All patients } \\
(\mathbf{n = 1 0 0 )}\end{array}$ & $\begin{array}{c}\text { TThN } \\
(\mathbf{n}=\mathbf{2 3})\end{array}$ & $\begin{array}{c}\text { TThE } \\
(\mathbf{n}=77)\end{array}$ \\
\hline Age, mean (SD) & $52.8(9.0)$ & $52.7(11.6)$ & $52.9(8.2)$ \\
\hline Weight, kg mean (SD) & $102.7(24.6)$ & $104.0(20.4)$ & $102.3(25.8)$ \\
\hline Height, cm mean (SD) & $176.4(6.3)$ & $176.0(7.7)$ & $176.6(5.9)$ \\
\hline BMI, kg/m² mean (SD) & $32.9(7.6)$ & $33.5(6.0)$ & $32.8(8.0)$ \\
\hline Age of hypogonadism diagnosis, mean (SD) & $49.3(9.6)$ & $51.4(11.1)$ & $48.7(9.0)$ \\
\hline Baseline/historical TT, nmol/L mean (SD) & $6.9(2.6)$ & $6.6(2.1)$ & $7.0(2.8)$ \\
\hline Race, n (\%) & & & \\
\hline Caucasian & $87(87.0)$ & $20(87.0)$ & $67(87.0)$ \\
\hline Black & $1(1.0)$ & $0(0.0)$ & $1(1.3)$ \\
\hline Asian & $5(5.0)$ & $1(4.3)$ & $4(5.2)$ \\
\hline Hispanic & $1(1.0)$ & $0(0.0)$ & $1(1.3)$ \\
\hline Middle-Eastern & $5(5.0)$ & $1(4.3)$ & $4(5.2)$ \\
\hline Other & $1(1.0)$ & $1(4.3)$ & $0(0.0)$ \\
\hline
\end{tabular}

BMI: body mass index; ITT: intent to treat; SD: standard deviation; TT: testosterone therapy; TThE: testosterone therapy-experienced; TThN: testosterone therapy-naive. 


\begin{tabular}{|c|c|c|c|c|c|c|c|c|c|c|c|c|c|c|c|c|}
\hline \multirow{3}{*}{ Cohort } & \multirow{3}{*}{$\mathbf{n}$} & \multicolumn{15}{|c|}{ Domain } \\
\hline & & \multicolumn{5}{|c|}{ Effectiveness } & \multicolumn{5}{|c|}{ Convenience } & \multicolumn{5}{|c|}{ Global satisfaction } \\
\hline & & Do & D30 & D60 & D90 & EP & D0 & D30 & D60 & D90 & EP & Do & D30 & D60 & D90 & EP \\
\hline TThE bid & 52 & 44.9 & $51.6^{*}$ & $52.9^{*}$ & $52.8^{*}$ & NA & 56.7 & $70.6^{*}$ & $71.8^{*}$ & $73.1^{*}$ & NA & 50.4 & 51.5 & 49.0 & 47.9 & NA \\
\hline TThE tid & 17 & 37.3 & $53.1^{*}$ & $52.6^{*}$ & $50.0^{*}$ & $59.2^{*}$ & 56.9 & $73.9^{*}$ & $76.1^{*}$ & $76.8^{*}$ & $76.1^{*}$ & 40.8 & 50.8 & $53.4^{*}$ & $53.4^{*}$ & $52.9^{*}$ \\
\hline TThE all & 69 & 43.0 & $52.0^{*}$ & $52.8^{*}$ & $52.1^{*}$ & $54.3^{*}$ & 56.8 & $71.4^{*}$ & $72.9^{*}$ & $74.0^{*}$ & $73.8^{*}$ & 48.0 & 51.3 & 50.1 & 49.3 & 49.2 \\
\hline TThN bid. & 10 & NA & 58.3 & 57.2 & 65.0 & NA & NA & 63.9 & 66.7 & 67.8 & NA & NA & 57.1 & 55.7 & 60.0 & NA \\
\hline TThN tid & 13 & NA & 44.0 & 44.9 & 41.0 & 45.3 & NA & 73.5 & 79.1 & 76.9 & 75.6 & NA & 52.2 & 52.2 & 45.1 & 46.7 \\
\hline TThN all & 23 & NA & 50.2 & 50.2 & 51.4 & 53.9 & NA & 6.93 & 73.7 & 72.9 & 72.2 & NA & 54.3 & 53.7 & 51.6 & 52.5 \\
\hline
\end{tabular}

${ }^{*}$ Statistically significant change from day 0 ( $\left.\mathrm{p}<0.05\right)$. bid: twice daily; EP: endpoint (value at the last visit study visit [D90 or D120]); NA: not applicable; tid: three times daily; TThE: testosterone therapy-experienced; TThN: testosterone therapy-naive. 


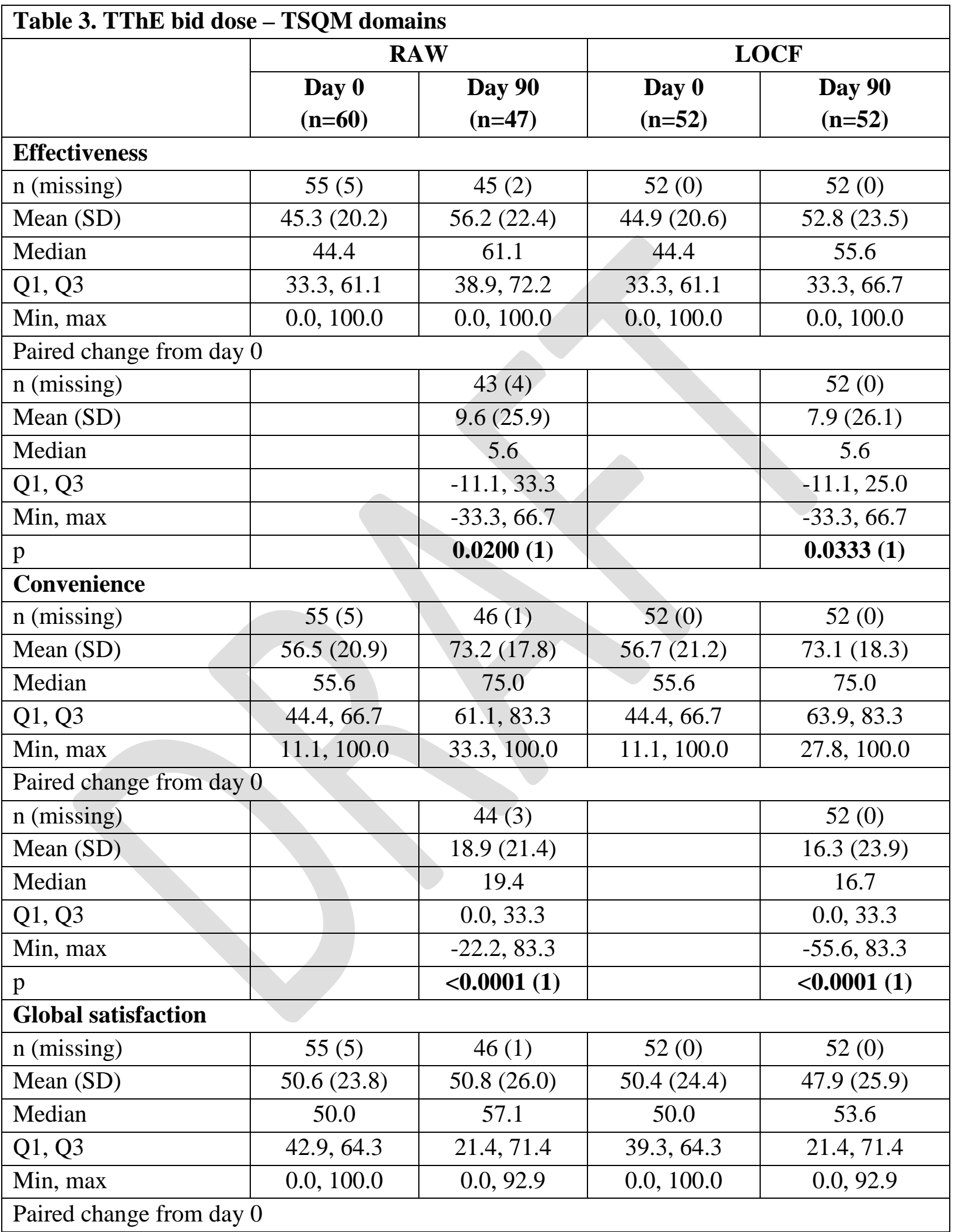




\begin{tabular}{|l|c|c|c|c|}
\hline $\mathrm{n}$ (missing) & & $44(3)$ & & $52(0)$ \\
\hline Mean (SD) & & $-0.6(33.0)$ & & $-2.5(31.9)$ \\
\hline Median & & -7.1 & & -7.1 \\
\hline Q1, Q3 & & $-25.0,17.9$ & & $-25.0,14.3$ \\
\hline Min, max & & $-64.3,85.7$ & & $-64.3,85.7$ \\
\hline p & & $\mathbf{0 . 8 9 6 6 ~ ( 1 )}$ & & $\mathbf{0 . 5 7 8 8 ~ ( 1 )}$ \\
\hline
\end{tabular}

LOCF: last observation carried forward; SD: standard deviation; TSQM: Treatment Satisfaction Questionnaire for Medication; TThE: testosterone therapy-experienced.

Table 4. Most important symptom for all patients $(n=92)$

\begin{tabular}{|l|c|}
\hline & $\mathrm{n}(\%)$ \\
\hline Difficulty achieving/maintaining an erection & $26(28.3)$ \\
\hline Low sex drive & $23(25.0)$ \\
\hline Fatigue/loss of energy & $23(25.0)$ \\
\hline Mood changes/irritability & $5(5.4)$ \\
\hline Increased body fat & $5(5.4)$ \\
\hline Decline in general feeling of well-being & $3(3.3)$ \\
\hline Depression/depressed mood & $3(3.3)$ \\
\hline Difficulty falling asleep/staying asleep & $2(2.2)$ \\
\hline Inability to concentrate & $1(1.1)$ \\
\hline Decreased physical activity/vitality & $1(1.1)$ \\
\hline
\end{tabular}




\begin{tabular}{|l|c|c|}
\hline \multicolumn{2}{|c|}{ Table 5. Side-by-side comparison of TTh topical products } \\
\hline $\begin{array}{l}\text { Testosterone per day } \\
\text { (starting dose) }\end{array}$ & TNG & Topical transdermal gel \\
\hline Delivery mechanism & Nasal applicator & $\begin{array}{c}50 \mathrm{mg} \text { androgel }{ }^{26} \\
50 \mathrm{mg} \text { testim }\end{array}$ \\
\hline Black box warning & None & $\begin{array}{c}\text { Spread by hand to upper arms/shoulders and/or right } \\
\text { and left abdomen }\end{array}$ \\
\hline Applications per day & 2 or 3 & Transference risk \\
\hline $\begin{array}{l}\text { Time required for a single } \\
\text { application }\end{array}$ & 10 seconds & 8-10 minutes \\
\hline Application notes & NA & $\begin{array}{c}\text { Includes application, drying and estimated cleanup } \\
\text { times required to reduce transference risks } \\
\text { Area must be continually covered and/or product } \\
\text { removed if skin-to-skin contact with treated area and } \\
\text { another person is anticipated }\end{array}$ \\
\hline
\end{tabular}




\section{References}

1 Men’s Health Review Panel. Men’s Health Guidelines for Family Medicine. 1st ed. MUMS Guideline Clearinghouse; 2017.

2 Wang C, Cunningham G, Dobs A, et al. Long-term testosterone gel (AndroGel) treatment maintains beneficial effects on sexual function and mood, lean and fat mass, and bone mineral density in hypogonadal men. J Clin Endocrinol Metab 2004 May;89(5):2085-98.

3 Dean JD, Carnegie C, Rodzvilla J, et al. Long-term effects of testim(r) 1\% testosterone gel in hypogonadal men. Rev Urol 2005 Spring;7(2):87-94.

4 Pastuszak A, Gomez L, Scovell J, et al. Comparison of the Effects of Testosterone Gels, Injections, and Pellets on Serum Hormones, Erythrocytosis, Lipids, and Prostate-Specific Antigen. Sex Med 2015 Sep;3(3):165-73.

5 Zhang GY, Gu YQ, Wang XH, et al. A pharmacokinetic study of injectable testosterone undecanoate in hypogonadal men. J Androl 1998 Nov-Dec;19(6):761-8.

6 Hajjar RR, Kaiser FE, Morley JE. Outcomes of long-term testosterone replacement in older hypogonadal males: a retrospective analysis. J Clin Endocrinol Metab 1997 Nov;82(11):37936.

7 Malkin CJ, Pugh PJ, West JN, et al. Testosterone therapy in men with moderate severity heart failure: a double-blind randomized placebo controlled trial. Eur Heart J 2006 Jan;27(1):57-64.

8 Kühnert B, Byrne M, Simoni M, et al. Testosterone substitution with a new transdermal, hydroalcoholic gel applied to scrotal or non-scrotal skin: a multicentre trial. Eur J Endocrinol. 2005 Aug;153(2):317-26.

9 NATESTO ${ }^{\circledR}$ (Testosterone Nasal Gel 4.5\%) Product Monograph, Acerus Biopharma Inc. November 27, 2017.

${ }^{10}$ Ohdo S. Chronopharmacology focused on biological clock. Drug Metab Pharmacokin 2007; 22(1):3-14.

${ }^{11}$ Kriegsfeld LJ, Silver R. The regulation of neuroendocrine function: timing is everything. Horm Behav 2006;49(5):557-74.

${ }^{12}$ Lincoln GA, Andersson H, Loudon A. Clock genes in calendar cells as the basis of annual timekeeping in mammals- a unifying hypothesis. J Endocrinol 2003;179(1):1-13.

${ }^{13}$ Rogol AD, Tkachenko N and Bryson N. Natesto ${ }^{\circledR}$, a novel testosterone nasal gel, normalizes androgen levels in hypogonadal men. Andrology 2016 Jan;4(1):46-54.

${ }^{14}$ Ramasamy, R. Effects of NATESTO ${ }^{\circledR}$ on Reproductive Hormones and Semen Parameters: A Prospective Clinical Trial. Paper presented at: American Society for Reproductive Medicine (ASRM); Oct 2018; Denver, CO, USA.

15 Mohamed O, Freundlich RE, Dakik HK, et al. The quantitative ADAM questionnaire: a new tool in quantifying the severity of hypogonadism. International Journal of Impotence Research 2010;22(1):20-4.

${ }^{16}$ Bharmal M, Payne K, Atkinson MJ, et al. Validation of an abbreviated Treatment Satisfaction Questionnaire for Medication (TSQM-9) among patients on antihypertensive medications. HRQOL 2009 27;7:36. 
${ }^{17}$ Kovac JR, Rajanahally S, Smith RP, et al. Patient satisfaction with testosterone replacement therapies: the reasons behind the choices. J Sex Med 2014 Feb;11(2):553-62.

${ }^{18}$ Baillargeon J, Urban RJ, Ottenbacher KJ, et al. Trends in androgen prescribing in the United States, 2001 to 2011. JAMA Intern Med 2013;173:1465-6.

${ }^{19}$ Snyder CF, Aaronson NK, Choucair AK, et al. Implementing patient-reported outcomes assessment in clinical practice: a review of the options and considerations. Qual Life Res 2012; 21:1305-14.

${ }^{20}$ Selby JV, Beal AC, Frank L. The Patient-Centered Outcomes Research Institute (PCORI) national priorities for research and initial research agenda. JAMA 2012;307:1583-84.

${ }^{21}$ Valderas JM, Kotzeva A, Espallargues M, et al. The impact of measuring patient-reported outcomes in clinical practice: a systematic review of the literature. Qual Life Res 2008; 17:179-93.

${ }^{22}$ Gelhorn HL, Bodhani AR, Wahala LS, et al. Development of the Hypogonadism Impact of Symptoms Questionnaire Short Form: Qualitative Research. J Sex Med 2016 Nov;13(11):1729-36.

${ }^{23}$ Cobden DS, Niessen LW, Barr CE, Rutten FF, Redekop WK (2010) Relationships among selfmanagement, patient perceptions of care, and health economic outcomes for decision-making and clinical practice in type 2 diabetes. Value Health 13: 138-47.

${ }^{24}$ Pollack MF, Purayidathil FW, Bolge SC, Williams SA (2010) Patient-reported tolerability issues with oral antidiabetic agents: Associations with adherence; treatment satisfaction and health-related quality of life. Diabetes Res Clin Pract 87: 204-10.

${ }^{25}$ Biderman A, Noff E, Harris SB, Friedman N, Levy A (2009) Treatment satisfaction of diabetic patients: what are the contributing factors? Fam Pract 26:102-8.

${ }^{26}$ ANDROGEL (testosterone gel) Product Monograph. BGP Pharma ULC. Canada. January 8, 2016.

27 TESTIM (testosterone gel) Product Monograph. Paladin Labs, Inc. Canada. February 3, 2017. 


\section{Selection of Study Population}

The trial enrolled 117 male participants ( 24 treatment-naïve and 93 previous topical TRT users) at 11 investigative sites in Canada. Volunteers were to be admitted into the study after signing the ICF, having met all of the inclusion criteria and none of the exclusion criteria (detailed below). These could be provided as supplemental material, if the reviewers believe this would be useful.

\section{Inclusion Criteria}

Participants who met all of the following inclusion criteria were eligible for participation in the study:

1. Hypogonadal male between 18 and 65 years of age, inclusive;

2. Able to understand and provide signed informed consent;

3. Have documented total serum testosterone levels $\leq 300 \mathrm{ng} / \mathrm{dL} / 10.4 \mathrm{nmol} / \mathrm{L}$;

4. Are currently being treated with any form of a topical testosterone replacement therapy for at least three months, or are treatment-naive.

\section{Exclusion Criteria}

Participants who met any of the following criteria were excluded from participation in the study:

1. In the opinion of the Investigator, significant intercurrent disease of any type, in particular liver, kidney, heart disease, or psychiatric illness;

2. History of pituitary or hypothalamic tumors or history of any malignancy (including breast and prostate cancers) within the past 5 years and excluding basal cell or squamous cell carcinoma of the skin curatively treated by surgery;

3. Prostatomegaly or history of abnormal PSA levels $(>10.0 \mathrm{ng} / \mathrm{mL})$. If PSA is $>10 \mathrm{ng} / \mathrm{mL}$, a recent negative biopsy must be documented (within the last 12 months);

4. History of nasal disorders, nasal or sinus surgery, nasal fracture within the previous 6 months or nasal fracture that caused a deviated anterior nasal septum surgery, mucosal inflammatory disorders, specifically Sjogren's syndrome;

5. Use of any form of intranasal medication delivery other than periodic short-term (less than 3 days) use of sympathomimetic decongestants;

6. History of severe adverse drug reactions to testosterone therapies;

7. History or current evidence of abuse of alcohol or any drug substance;

8. Current treatment with other androgens (e.g., dehydroepiandrosterone [DHEA]), anabolic steroids, or other sex hormones;

9. Treatment with estrogens, gonadotropin-releasing hormone $(\mathrm{GnRH})$ agonists, or growth hormone within the previous 12 months;

10. Treatment with drugs that interfere with the metabolism of testosterone, such as anastrozole, clomiphene, dutasteride, finasteride, flutamide, ketoconazole, spironolactone, or testolactone;

11. Poor compliance history;

12. Participation in any other research study during the conduct of this study or 30 days prior to the initiation of this study. 